\title{
High expression of microRNA-200c predicts poor clinical outcome in diffuse large B-cell lymphoma
}

\author{
MATTIAS BERGLUND ${ }^{1}$, GUSTAV HEDSTRÖM ${ }^{1}$, ROSE-MARIE AMINI ${ }^{2}$, \\ GUNILLA ENBLAD $^{1}$ and ULF THUNBERG ${ }^{1}$ \\ Departments of ${ }^{1}$ Radiology, Oncology and Radiation Sciences, \\ ${ }^{2}$ Immunology, Genetics and Pathology, Uppsala University, Uppsala, Sweden
}

Received October 9, 2012; Accepted November 12, 2012

DOI: $10.3892 /$ or.2012.2173

\begin{abstract}
Diffuse large B-cell lymphoma (DLBCL) is a heterogeneous group of B-cell lymphomas. A new and important tool for understanding the biology and clinical course of DLBCL is microRNA expression. This study presents microRNA-200c expression data from 61 DLBCL patients treated with CHOP or R-CHOP. Patients with high microRNA-200c expression had a median survival of 20.3 months and a significantly shorter overall survival $(\mathrm{P}=0.019)$ compared to patients with low microRNA-200c expression, who had a median survival of 35.8 months. We also found that patients treated with R-CHOP only and displaying high microRNA-200c expression had a significantly shorter overall survival compared to patients with low microRNA-200c expression, where all patients were still alive at the time of the last follow-up $(\mathrm{P}=0.0036)$. Lastly, we found that patients with high microRNA-200c expression had a significantly shorter time from initial diagnosis to the first relapse compared to patients with low microRNA-200c expression $(\mathrm{P}=0.0001)$. To our knowledge, this is the first study showing that the expression of microRNA-200c affects the clinical outcome of DLBCL patients, and that microRNA$200 \mathrm{c}$ is involved in the biology of DLBCL development, although larger studies are necessary to confirm this. Further investigations may also help to elucidate the biological role of microRNA-200c in DLBCL.
\end{abstract}

\section{Introduction}

Diffuse large B-cell lymphomas (DLBCL) essentially encompass a heterogeneous group of B-cell lymphomas which typically have an aggressive clinical course. For many years CHOP (cyclophosphamide, adriamycin, vincristine and prednisone) was used as the standard treatment for DLBCL

Correspondence to: Dr Ulf Thunberg, Department of Radiology, Oncology and Radiation Sciences, Rudbeck Laboratory, Uppsala University, SE-751 85 Uppsala, Sweden

E-mail: ulf.thunberg@onkologi.uu.se

Key words: microRNA-200c, diffuse large B-cell lymphoma, prognosis patients, however by adding rituximab (CD20 antibody) to this regime, prognosis has improved significantly (1).

The most commonly used prognostic tool in DLBCL is the International Prognostic Index (IPI), which takes into account clinical parameters such as age, clinical stage and performance status. Nonetheless, many studies have identified certain molecular markers of prognostic relevance, such as the classification of DLBCL as germinal center B-like (GC) or activated $\mathrm{B}$ cell-like (ABC) groups using expression profiling techniques $(2,3)$. In addition, many individual biomarkers have been identified and some are prognostic even in the rituximab era (4).

Recently, microRNA (miRNA) expression has been shown to be important in understanding the biology and clinical course of DLBCL, as well as other tumors. miRNAs are endogenous small noncoding RNAs, approximately 22 nucleotides in length (5). In mammals, miRNAs mediate translational suppression by binding to the 3'UTR of messenger-RNAs (6). miRNAs have been shown to be involved in many cellular processes such as differentiation and apoptosis, as well as tumorigenesis $(7,8)$. Furthermore, miRNAs have been reported to predict outcome in many different tumor types (9-14).

One miRNA of interest is miR-200c, which is activated by $\mathrm{p} 53$. This activation of miR-200c is accomplished by p53-binding to the miR-200c promoter (15). When miR-200c is upregulated it has been found to affect three different pathways.

The first pathway involves the inhibition of ZEB1 and ZEB2, transcriptional repressors of E-cadherin which is an epithelial marker $(16,17)$. This inhibition of the repressors leads to higher expression of E-cadherin and probably contributes to less cancer progression and metastasis by hampering the tumor and epithelial-to-mesenchymal transition (EMTs), which has been shown to occur in metastatic cancer cells $(16,17)$. It has also been shown in DLBCL that GC-subtype patients, who often have longer survival compared to ABC-subtype patients, express lower amounts of ZEB1 compared to the ABC-subtype (18). Of note, this low expression of ZEB1 in the GC-subtype was also correlated with high expression of BCL-6, a zinc finger transcription factor that is highly expressed in normal germinal centre B-cells (18). It has also been shown that patients with high expression of BCL-6 have longer survival than patients with relatively low expression of BCL-6 in DLBCL (19). 
miR-200c is also involved in the regulatory pathway of BMI1; when miR-200c is upregulated it leads to a downregulation of BMI1, which is a protein that maintains cell stemness properties $(20,21)$. By negating the cell stemness properties through induction of high miR-200c expression, it has been showed that clonal expansion of breast cancer cells is inhibited, while the growth of embryonal carcinoma cells in vitro is suppressed by high expression of miR-200c (20).

However, while both of these studies purport that high expression of miR-200c would be of benefit for cancer patients in terms of survival, recently, evidence of a third alternative pathway has been found which shows that breast cancer patients with high expression of miR-200s actually have a worse outcome compared to patients with low expression (22). Korpal et al showed that high levels of miR-200s inhibit Sec23a and the secretory pathway which leads to a downregulation of metastasissuppressive proteins such as insulin-like growth factor binding protein 4 (Igfbp4) and tubulointerstital nephritis antigen-like 1 (Tinagl 1). These findings indicate a possible mechanism by which a higher mesenchymal-to-epithelial transition (MET) rate occurs, a feature typically found in metastasis (22).

Moreover, it has also been shown that high expression of miR-200c induces chemoresistance in esophageal cancer leading to a shorter survival for those patients expressing high levels of miR-200c (23). This chemoresistance is probably caused by miR-200c-induced downregulation of PPP2R1B expression, a subunit of protein phosphatase $2 \mathrm{~A}$, which results in activation of Akt signalling, known to be involved in chemoresistance in various cancers (23-26). Noteworthy, it has been shown that DLBCL patients with high p-AKT expression displayed a poorer survival compared to those with low p-AKT expression (26).

In the present study, we investigated the expression levels of miR-200c in 61 de novo DLBCL cases by reverse transcriptase-PCR. The miR-200c expression levels were compared to clinical features, including overall survival and GC subtype. We also compared the expression of miR-200c in tumor tissue, to that in surrounding tissue and normal controls.

\section{Materials and methods}

Diagnostic lymph nodes from 61 patients with de novo DLBCL were included in the study. The clinical characteristics for the patients for whom a Pfaffl-value was obtained are presented in Table I. We also used 13 normal lymph nodes as controls. All DLBCL biopsies were evaluated by a pathologist, and the areas with the most typical lymphoma tissue were marked. After the evaluation, the areas with typical lymphoma tissue and the 'surrounding tissue', with a more normal morphology, were microdissected with a scalpel into two parts: 'tumor tissue' and 'surrounding tissue'.

Total miRNA was extracted from the dissected tissue using Recoverall Total Nucleic Acid Isolation (Ambion, Austin, TX, USA) according to the standard protocol. For the reverse transcriptase reaction, $2 \mu \mathrm{l}$ or $\sim 5 \mathrm{ng}$ of RNA was used, and the amplification was carried out in a Gene Amp 9700 PCR machine (Applied Biosystems, CA, USA) according to the manufacturers protocol.

Primers for specific reverse transcription of miR-200c (Applied Biosystems) were used to create cDNA. RNU6b, a
Table I. Clinical features of the patients with a known miR-200c value.

\begin{tabular}{|c|c|c|c|}
\hline & $\begin{array}{c}\text { Low } \\
\text { miR-200c } \\
\text { (n) }\end{array}$ & $\begin{array}{c}\text { High } \\
\text { miR-200c } \\
\text { (n) }\end{array}$ & P-value \\
\hline Total patients & 34 & 27 & \\
\hline \multicolumn{4}{|l|}{ Age, years } \\
\hline Mean & 63 & 69 & $0.1^{\mathrm{a}}$ \\
\hline Median & 64.5 & 69 & \\
\hline Range & $17-90$ & $37-88$ & \\
\hline Gender & & & $0.59^{\mathrm{b}}$ \\
\hline Females & 10 & 10 & \\
\hline Males & 24 & 17 & \\
\hline Stage & & & $0.43^{\mathrm{b}}$ \\
\hline I-II & 15 & 10 & \\
\hline III-IV & 15 & 16 & \\
\hline NA & 4 & 1 & \\
\hline aaIPI & & & $0.41^{\mathrm{b}}$ \\
\hline $0-1$ & 20 & 14 & \\
\hline $2-3$ & 10 & 12 & \\
\hline NA & 4 & 1 & \\
\hline CHOP-like treatment & & & $1^{\mathrm{b}}$ \\
\hline Yes & 24 & 18 & \\
\hline No & 3 & 3 & \\
\hline NA & 7 & 6 & \\
\hline Rituximab & & & $0.06^{\mathrm{b}}$ \\
\hline Yes & 17 & 8 & \\
\hline No & 12 & 17 & \\
\hline NA & 5 & 2 & \\
\hline GC/non-GC & & & $0.43^{\mathrm{b}}$ \\
\hline $\mathrm{GC}$ & 12 & 13 & \\
\hline Non-GC & 19 & 13 & \\
\hline NA & 3 & 1 & \\
\hline
\end{tabular}

aaIPI, age adjusted IPI; CHOP-like refers to CHOP (cyclophosphamide, adriamycin, vincristine and prednisone) or a CHOP-like regimen; GC, germinal centre-derived lymphoma; NA, not applicable. ${ }^{a}$ Unpaired t-test (two-sided). ${ }^{\mathrm{b}}$ Fisher exact test (two-sided).

housekeeping gene, was used as a reference. In the quantification of miRNA, all sample reactions were performed in triplicate and all amplifications were carried out in a TaqMan Real-Time PCR machine (Applied Biosystems), as described by the manufacturer.

The relative expression of miR-200c compared to RNU6b was calculated using a modified Pfaffl method due to the fact that no controls were available from each corresponding DLBCL (27). Our modified Pfaffl method was as follows: $\log 2$ $\left[\left(\right.\right.$ Efficiencies of RNU6b $\left.{ }^{\text {Cycle number of RNU6b }}\right) /($ Efficiencies of miR$\left.\left.200 c^{\text {Cycle number of miR-200c }}\right)\right]$.

The patients were assigned to GC or non-GC categories based on immunohistochemistry according to the algorithm 


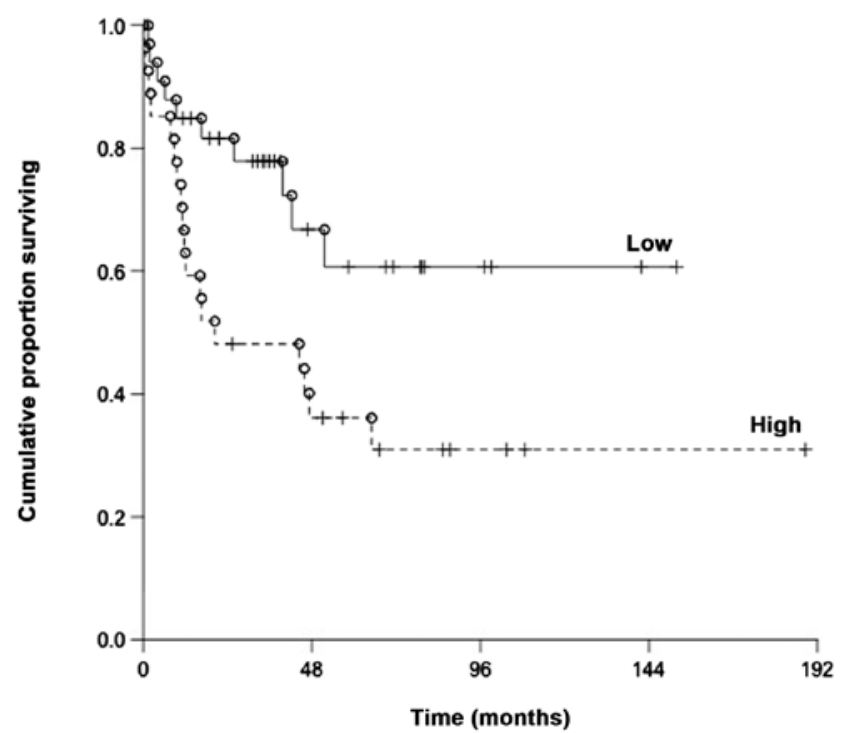

Figure 1. miR-200c expression and overall survival in DLBCL patients $(\mathrm{P}=0.019)$. The patients of the cohort were divided into cases with low and high expression of miR-200c based on the mean Pfaffl-value for miR-200c. The P-value was evaluated by a log-rank test.

presented by Hans et al (28). The clinical information was obtained from patient records. The study was approved by the Ethics Committee of Uppsala University.

Statistical methods. To calculate whether the relative expression of miR-200c was of any prognostic importance, the values of relative expression were divided in to two groups according to the mean and median values. The overall survival rates of the groups of DLBCL patients with either low or high relative expression of miR-200c were compared by Kaplan-Meier survival analysis and log-rank test to investigate the prognostic importance of miR-200c expression. Overall survival was calculated from the date of diagnosis until the last follow-up or death.

To evaluate whether there were any differences in the relative expression of miR-200c in the tumor tissues, the surrounding tissue and the normal controls, we compared the three groups pairwise using a t-test. To determine the differences in (mean) age at diagnosis between groups with high versus low expression of miR-200c, an unpaired t-test was performed. A Fisher's exact test was used to examine for differences in proportions between the same groups regarding different clinical parameters (Table I).

All statistical analyses were performed using Statistica 10.0 (StatSoft, Tusla, OK, USA) and the R statistical program (http://www.r-project.org/). Probabilities of $<0.05$ were judged as an indication of statistical significance.

\section{Results}

Twenty-seven (44\%) patients had high relative expression (above the mean; mean Pfaffl-value of -6.248818) and 34 (56\%) patients had low relative expression of miR-200c (below the mean).

The DLBCL patients with high relative expression had a significantly shorter overall survival as compared to those

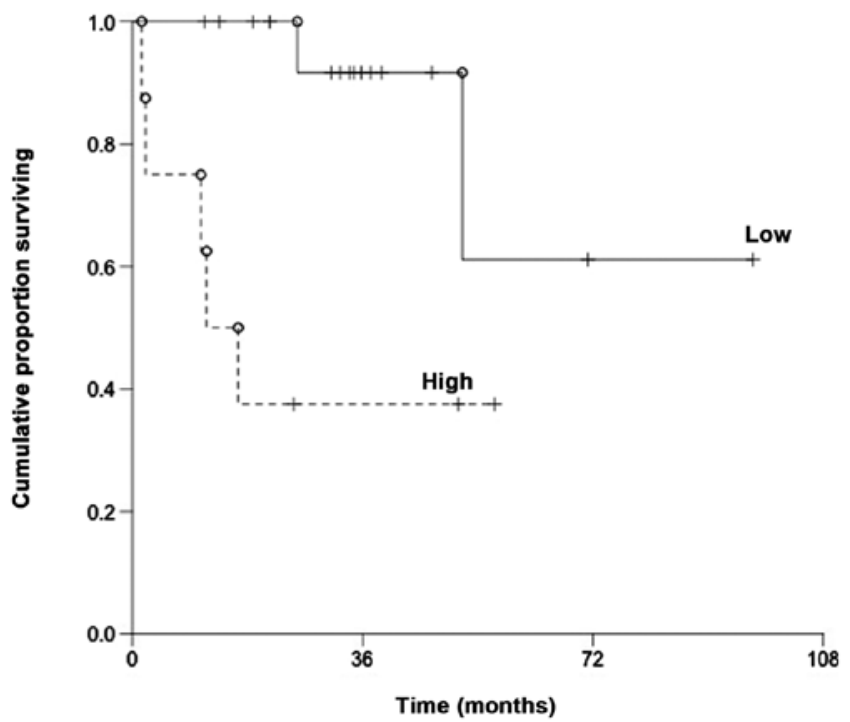

Figure 2. Overall survival of DLBCL patients treated with R-CHOP or R-CHOP-like regimen and miR-200c expression $(\mathrm{P}=0.0036)$. The patients of the cohort were divided into cases with low and high expression of miR-200c based on the mean Pfaffl-value for miR-200c. The P-value was evaluated by a log-rank test.

with a low expression; with a median of 20.3 months and 35.8 months, respectively ( $\mathrm{P}=0.019)$ (Fig. 1).

The mean age at diagnosis was 69 years for patients with high expression of miR-200c and 63 years for patients with low expression of miR-200c. The median age at diagnosis was 68 years for patients with high expression of miR-200c and 63 years for patients with low expression. No statistically significant differences were found between the groups regarding age at diagnosis.

For patients treated with R-CHOP $(n=25)$, there was a statistically significant difference $(\mathrm{P}=0.0036)$ in overall survival between patients with high relative expression of miR-200c (above the mean) and low relative expression of miR-200c (below the mean) (Fig. 2).

Patients with high relative expression of miR-200c had a $50 \%$ survival at 20.3 months whereas 15 patients with low relative expression of miR-200c were still alive at the last follow-up date. For patients not treated with R-CHOP (n=29), no difference in overall survival was found $(\mathrm{P}=0.35)$.

When measuring the time from initial diagnosis to the first relapse we found a clear difference where early relapsing patients expressed high miR-200c to a greater extent than patients with low miR-200c expression ( $\mathrm{P}=0.0001)$ (Fig. 3).

There were no differences between the high or low miR-200c expression groups in terms of age, IPI and stage, either when the groups were determined by mean (Table I) or median value of relative miR-200c expression. There was no statistically significant difference in survival between the genders $(\mathrm{P}=0.59)$ in either the $\mathrm{GC}$ or the non-GC subtype $(\mathrm{P}=0.43)$.

When the tumor tissue, surrounding tissue and normal lymph nodes were compared in terms of miR-200c expression, no significant differences were observed between them (normal lymph nodes: mean Pfaffl-value of -6.244954, surrounding 


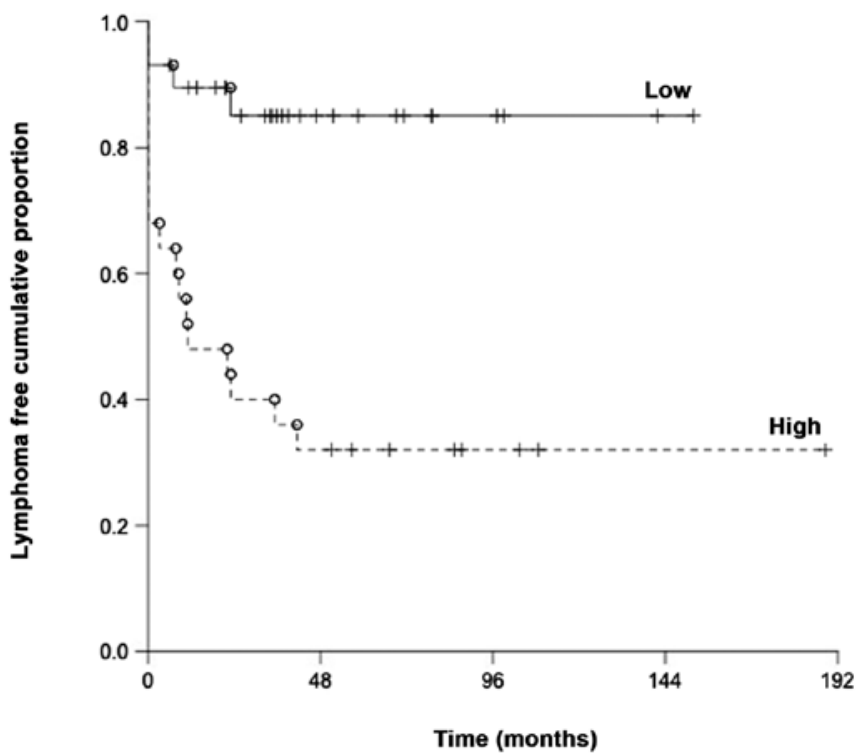

Figure 3. Time to relapse and miR-200c expression $(\mathrm{P}=0.0001)$. Patients were divided into cases with low and high expression of miR-200c based on the mean Pfaffl-value for miR-200c. Time was measured from diagnosis to first relapse. For patients without complete response, time from diagnosis to relapse were set to 0 . The P-value was evaluated by a log-rank test.

tissue: mean Pfaffl-value of -6.30193 and tumor tissue: mean Pfaffl-value of -6.248818).

\section{Discussion}

miR-200c has not been well studied in lymphomas; however, it has been shown that the GC-subtype of DLBCL expresses low amounts of the protein ZEB1 (18). Of note, a low expression of ZEB1 correlates with a high expression of miR-200c and taking into account the fact that GC-subtype patients have a proven longer survival compared to the ABC-subtype, it is tempting to hypothesize that high expression of miR-200c leads to a significantly better survival (16-19).

However, our results instead indicated the opposite trend, where patients with high expression of miR-200c showed a significantly worse outcome compared to patients with low expression of miR-200c $(\mathrm{P}=0.019)$. This finding was rather remarkable and somewhat unexpected. This correlation could, of course, be explained by the relatively low sample size in our study, but it would be rather unfortunate if our results are in total disagreement with the true biological impact. We did not find any connection between the expression of miR-200c and the GC-subtype or non-GC-subtype $(\mathrm{P}=0.37)$ indicating that the expression of miR-200c is probably independent of DLBCL subtype. This finding was also rather unexpected considering that high expression of miR-200c leads to low expression of ZEB1 and low expression of ZEB1 has been reported for the GC-subtype (16-19).

However, considering that our data indicate that high miR-200c expression correlates with a significantly poorer outcome for DLBCL patients, it is tempting to speculate that the development of DLBCL and the biology of the tumor is to some extent explained by Korpal et al findings. They reported that breast cancer patients with high expression of miR-200s actually had a worse outcome compared to patients with low expression of miR-200s, which is in line with our results showing a worse outcome for DLBCL patients with high levels of miR-200c (22). According to Korpal et al, high levels of miR-200s inhibits Sec23a and the secretory pathway which lead to a downregulation of certain metastasis-suppressive proteins such as insulin-like growth factor binding protein 4 (Igfbp4) and tubulointerstitial nephritis antigen-like 1 (Tinagl 1) (22). This pathway can possibly lead to a higher mesenchymal-to-epithelial transition (MET), which has been shown to promote the development of metastasis (22).

Another plausible explanation for the correlation between high miR-200c expression and poor prognosis in DLBCL is that high miR-200c expression induces chemoresistance, which has been noted in esophageal cancer (23). Even though the chemotherapy for esophageal cancer and DLBCL is not the same, the finding that miR-200c activates Akt signaling in esophageal cancer fits well with the finding that DLBCL patients with high p-AKT expression have a poorer survival compared to patients with low p-AKT expression $(23,26)$. This explanation for miR-200c-induced chemoresistance can perhaps also be supported by our findings that DLBCL patients treated with R-CHOP and high miR-200c expression had a significantly shorter survival compared to patients treated with R-CHOP and low miR-200c expression $(\mathrm{P}=0.0036)$ and also that the time to relapse was significantly shorter for patients with high miR-200c $(\mathrm{P}=0.0001)$.

In summary, we showed that DLBCL patients with high expression of miR-200c have a significantly worse prognosis compared to DLBCL patients with low expression of miR-200c which can be seen in terms of overall survival particularly for DLBCL patients treated with R-CHOP. However, it is necessary to explore the biology behind this finding and further investigate the impact of chemoresistance in DLBCL to confirm our results.

\section{References}

1. Habermann TM, Weller EA, Morrison VA, et al: RituximabCHOP versus CHOP alone or with maintenance rituximab in older patients with diffuse large B-cell lymphoma. J Clin Oncol 24: 3121-3127, 2006.

2. Alizadeh AA, Eisen MB, Davis RE, et al: Distinct types of diffuse large B-cell lymphoma identified by gene expression profiling. Nature 403: 503-511, 2000.

3. Rosenwald A, Wright G, Chan WC, et al: The use of molecular profiling to predict survival after chemotherapy for diffuse largeB-cell lymphoma. N Engl J Med 346: 1937-1947, 2002.

4. Ninan MJ, Wadhwa PD and Gupta P: Prognostication of diffuse large B-cell lymphoma in the rituximab era. Leuk Lymphoma 52: 360-373, 2011.

5. Kim VN, Han J and Siomi MC: Biogenesis of small RNAs in animals. Nat Rev Mol Cell Biol 10: 126-139, 2009.

6. Valencia-Sanchez MA, Liu J, Hannon GJ and Parker R: Control of translation and mRNA degradation by miRNAs and siRNAs. Genes Dev 20: 515-524, 2006.

7. Davis BN and Hata A: microRNA in cancer: the involvement of aberrant microRNA biogenesis regulatory pathways. Genes Cancer 1: 1100-1114, 2010.

8. Hobert O: Common logic of transcription factor and microRNA action. Trends Biochem Sci 29: 462-468, 2004

9. Alencar AJ, Malumbres R, Kozloski GA, et al: MicroRNAs are independent predictors of outcome in diffuse large B-cell lymphoma patients treated with R-CHOP. Clin Cancer Res 17: 4125-41352011. 
10. Calin GA, Ferracin M, Cimmino A, et al: A microRNA signature associated with prognosis and progression in chronic lymphocytic leukemia. N Engl J Med 353: 1793-1801, 2005.

11. Marcucci G, Radmacher MD, Maharry K, et al: MicroRNA expression in cytogenetically normal acute myeloid leukemia. $\mathrm{N}$ Engl J Med 358: 1919-1928, 2008.

12. Montes-Moreno S, Martinez N, Sanchez-Espiridion B, et al: miRNA expression in diffuse large B-cell lymphoma treated with chemoimmunotherapy. Blood 118: 1034-1040, 2011.

13. Schwind S, Maharry K, Radmacher MD, et al: Prognostic significance of expression of a single microRNA, miR-181a, in cytogenetically normal acute myeloid leukemia: a Cancer and Leukemia Group B study. J Clin Oncol 28: 5257-5264, 2010.

14. Yanaihara N, Caplen N, Bowman E, et al: Unique microRNA molecular profiles in lung cancer diagnosis and prognosis. Cancer Cell 9: 189-198, 2006.

15. Chang $\mathrm{CJ}$, Chao $\mathrm{CH}$, Xia W, et al: p53 regulates epithelialmesenchymal transition and stem cell properties through modulating miRNAs. Nat Cell Biol 13: 317-323, 2011.

16. Korpal M,Lee ES, Hu G and Kang Y: The miR-200 family inhibits epithelial-mesenchymal transition and cancer cell migration by direct targeting of E-cadherin transcriptional repressors ZEB1 and ZEB2. J Biol Chem 283: 14910-14914, 2008.

17. Polyak K and Weinberg RA: Transitions between epithelial and mesenchymal states: acquisition of malignant and stem cell traits. Nat Rev Cancer 9: 265-273, 2009.

18. Papadopoulou V, Postigo A, Sanchez-Tillo E, Porter AC and Wagner SD: ZEB1 and CtBP form a repressive complex at a distal promoter element of the BCL6 locus. Biochem J 427: 541-550, 2010

19. Lossos IS, Jones CD, Warnke R, et al: Expression of a single gene, BCL-6, strongly predicts survival in patients with diffuse large B-cell lymphoma. Blood 98: 945-951, 2001.
20. Shimono Y, Zabala M, Cho RW, et al: Downregulation of miRNA-200c links breast cancer stem cells with normal stem cells. Cell 138: 592-603, 2009.

21. Wellner U, Schubert J, Burk UC, et al: The EMT-activator ZEB1 promotes tumorigenicity by repressing stemness-inhibiting microRNAs. Nat Cell Biol 11: 1487-1495, 2009.

22. Korpal M, Ell BJ, Buffa FM, et al: Direct targeting of Sec23a by miR-200s influences cancer cell secretome and promotes metastatic colonization. Nat Med 17: 1101-1108, 2011.

23. Hamano R, Miyata H, Yamasaki M, et al: Overexpression of miR-200c induces chemoresistance in esophageal cancers mediated through activation of the Akt signaling pathway. Clin Cancer Res 17: 3029-3038, 2011.

24. Liu LZ, Zhou XD, Qian G, Shi X, Fang J and Jiang BH: AKT1 amplification regulates cisplatin resistance in human lung cancer cells through the mammalian target of rapamycin/p70S6K1 pathway. Cancer Res 67: 6325-6332, 2007.

25. Thompson JE and Thompson CB: Putting the rap on Akt. J Clin Oncol 22: 4217-4226, 2004.

26. Uddin S, Hussain AR, Siraj AK, et al: Role of phosphatidylinositol 3'-kinase/AKT pathway in diffuse large B-cell lymphoma survival. Blood 108: 4178-4186, 2006.

27. Pfaffl MW: A new mathematical model for relative quantification in real-time RT-PCR. Nucleic Acids Res 29: e45, 2001.

28. Hans CP, Weisenburger DD, Greiner TC, et al: Confirmation of the molecular classification of diffuse large B-cell lymphoma by immunohistochemistry using a tissue microarray. Blood 103: 275-282, 2004 